\title{
Black hole ejections in mergers of small groups of galaxies
}

\author{
Pekka Heinämäki and Mauri Valtonen \\ Tuorla Observatory and Physics Department, University of Turku, \\ FIN-21500 Piikkiö, Finland
}

\begin{abstract}
.
The formation of a multiple black hole system via merging processes of galaxies is simulated. Starting from the hierarchical halo merger model by Lacey and Cole (1993) and the general assumption that supermassive black holes $\left(>10^{6} M_{\odot}\right)$ are common in the nuclei of galaxies we followed the formation of a four black hole system. Ultimately, instability of the four body system led to 835 symmetric two-sided slingshot ejections out of 10,000 simulations. This result was applied to the most straightforward application of the slingshot process, namely to the origin of extended radio sources.
\end{abstract}

\section{Introduction}

The present understanding of the formation of extended double radio sources (FR II) is far from clear. In particular, there are lot of puzzles which are not satisfactorily explained by the most popular so-called beam theory (Blandford and Rees 1974).

One difficulty is how to understand the process which collimates Mpc-scale jets from the scale of order one parsec and upwards. A free jet which is not confined by external pressure falls below the pressure of the ambient medium, at the latest, after a few kiloparsecs, at which point the jet should become unstable. Finally, lifetimes of the relativistic electrons are not long enough to produce the emission observed in the hot spots, such as the X-ray radiation in Cygnus A (Harris et al. 1994) or the optical radiation in 3C 33 (Meisenheimer et al. 1989). Obviously some re-acceleration mechanisms are needed. This is a problem in the beam theory in the case of a double hot spot in a single radio lobe (Lähteenmäki and Valtaoja 1999).

Another puzzle relates to the misalignment of the pc-scale and Mpc-scale jets. Appl et al. (1996) have found that more then 50 percent of double radio sources have misalignments of more then 45 degrees between these two scales.

Thus there is obviously a need to improve the established theories and to study alternative and additional theories. One solution may be the so-called slingshot theory. 


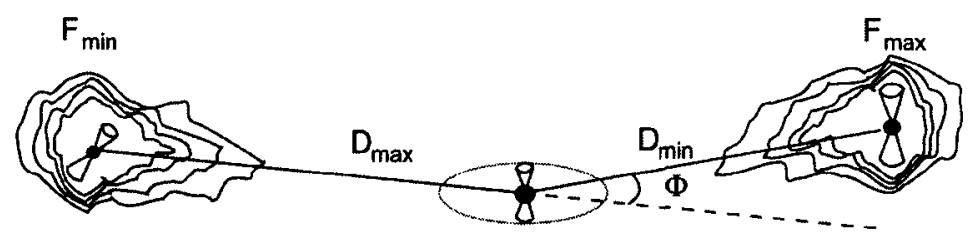

Figure 1. A schematic diagram of the central galaxy and extended radio lobes. The definitions of the parameters are illustrated. Black holes with twin beams are found in both radio lobes as well as in the nucleus of the central galaxy.

\section{Model and Simulations}

The general outline of the model can be divided into four stages. At the first stage, black hole masses and the merger times of their host galaxies were determined using an evolutionary model by Lacey and Cole (1993). Second, after two galaxies are presumed to have merged, the evolution of a binary black hole system starts under the influence of the gas and stars of the surrounding host galaxy. Third, two galaxies with binary black hole systems are presumed to have come together and the binary black hole systems start to approach each other in the nucleus of the merged galaxy. Fourth, a four black hole system is formed and the instability of this chaotic black hole system leads finally to mergers and ejections or escapes of the black holes.

When mergers between galaxies with black holes in both centers take place (on average once in a Gyr) dynamical friction drives black holes together in a relatively short timescale and a stable (lifetime more than one Gyr) black hole binary system may be produced in the new galaxy center. During this epoch three mechanisms, which shrink the orbits of the binary black holes, were taken into account: 1) stars which flow past the binary; 2) gravitational radiation; 3) cooling flow of gas. Adding black hole binary evolution to the hierarchical galaxy merger model finally led 30 percent of 10,000 simulations to end with a four black hole system. This provided an effective starting point for the slingshot process. About 25 percent of these four black hole systems ended up in two-sided ejections which are especially interesting when one studies double radio sources.

According to the slingshot process (Saslaw, Valtonen \& Aarseth 1974) black holes which escape from a galaxy can produce local emission in extended radio lobes which is comparable to the observed emission in different types of radio galaxies. Narrow weakly radiating filaments which connect the nucleus and the lobes are just tracks of the magnetic plasma which fast moving $\left(>1000 \mathrm{~km} \mathrm{~s}^{-1}\right)$ black holes have left in the surrounding medium (Valtaoja 1986, Valtonen 1999). Fig. 1 is a schematic picture of how the slingshot process produces a double radio source. The studied quantities are the bending angle $(\Phi)$, the distance ratio $\left(D_{r}=D_{\max } / D_{\min }\right)$ and the flux ratio $\left(F_{r}=F_{\max } / F_{\min }\right)$.

According to Valtaoja and Valtonen (1984) the total radio luminosity $P$ is a function of the distance of the radio lobe and of the ejection speed of the black 


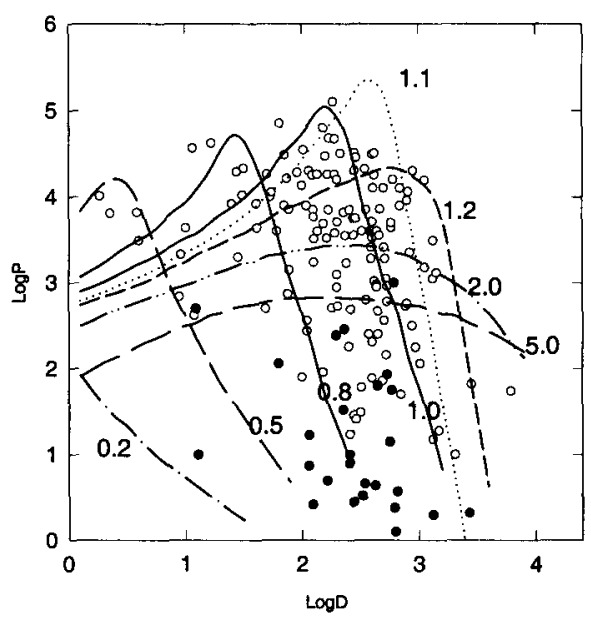

Figure 2. Total radio luminosity $P$ as a function of the distance of the radio lobe from the central galaxy. Open circles indicate FR II type radio sources and filled circles show the FR I type sources.

hole from the parent galaxy. Curves in Fig. 2 represent different escape velocities (1.0 is the velocity limit for an escape from the potential well of the parent galaxy) for the ejected black holes. In addition, we have plotted observations of radio galaxies in the complete sample of $3 \mathrm{C}$ radio sources. Open circles are FR II type radio sources and filled ones FR I type radio sources. Note that FR I sources could represent cases where the black hole escape was unsuccessful (ejection speed less than 1.0).

Using the curves shown in Fig. 2 we have calculated a flux-weighted 3$\mathrm{D}$ histogram for the distribution of the radio sources in the $\log F_{r}-\log D_{r}$ plane which is shown in Fig. 3. It reveals a separate peak at $\log D_{r} \simeq 0$ and $\log F_{r} \simeq 0$. We propose that this peak represents the "classical" double radio sources and that the cases with large $F_{r}$ represent sources for which the flux ratio is too large in order that they would be included in conventional radio galaxy samples.

Figs. 4 and 5 show the distributions of the bending angle $\Phi$ and the distance ratio $D_{r}$ for the simulations and for the sample of radio galaxies by Nilsson (1988).

For a more detail description, the reader may consult papers by Valtonen et al. (1994), Heinämäki (1998), Valtonen and Heinämäki (2000).

Acknowledgments. Special thanks to Kari Nilsson and Rami Rekola.

\section{References}

Appl, S., Sol, H. \& Vicente, L. 1996, A\&Ap, 310, 419 


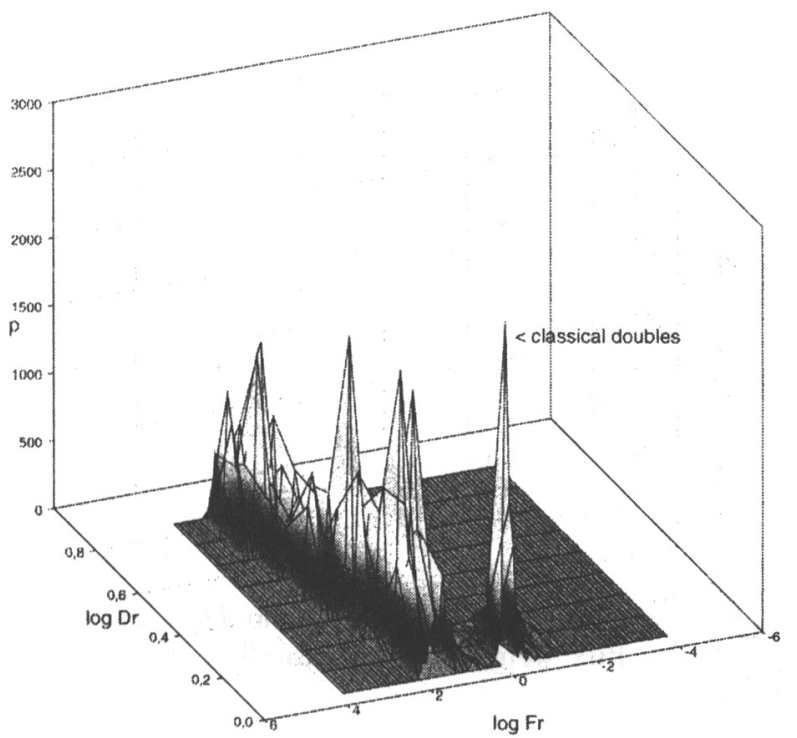

Figure 3. The surface plot of the 3-D histogram illustrates the flux ratio $F_{r}, D_{r}$ and weight parameter for the simulations. 


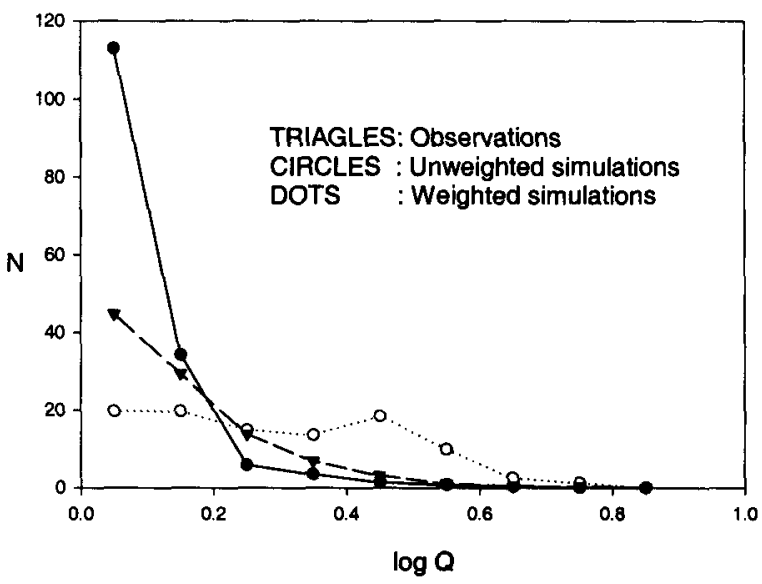

Figure 4. The distribution of the distance ratio $D_{r}$ for simulations (unweighted and with a flux weight suitable for flux limited samples and observations.

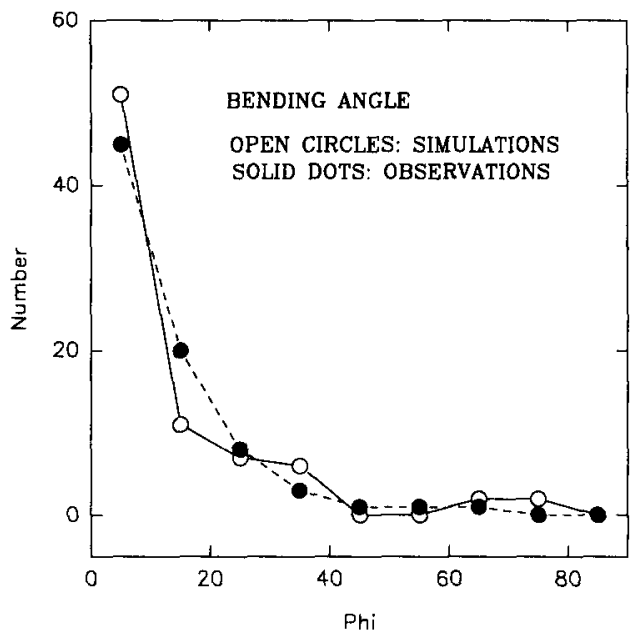

Figure 5. The distribution of the bending angle $\Phi$ for simulations and observations. 
Blandford, R. D., Rees, M.J. 1974, MNRAS, 169, 395

Harris, Carilli, C. L. and Perley, R. A. 1994, Nature, 367, 713

Heinämäki, P. 1998, PhD Thesis, Univ. of Turku

Lacey, C. and Cole, S. 1993, MNRAS, 262, 627

Lähteenmäki, A. and Valtaoja, E. 1999, AJ, 117, 1168

Meisenheimer, K., Röser, H-J., Hiltner, P. R., Yates, M. G., Longair, M. S., Chini, R. and Perley, R. A. 1989, A\&A, 219, 63

Nilsson, K. 1998, A\&AS, 132, 31

Valtaoja, E. and Valtonen, M. J. 1984, A\&A, 130, 373

Valtaoja, E. 1986, Ap\&SS, 127, 103

Valtonen, M. J. 1999, ApJ, 520, 97

Valtonen, M. J. and Heinämäki, P., 2000, Apj, 530, (to appear in Feb 10 edition)

Valtonen, M. J., Mikkola, S., Heinämäki, P. and Valtonen H. 1994, ApJS, 95, 69

Saslaw, W. C., Valtonen, M. J. and Aarseth, S. J. 1974, ApJ, 190, 253 